\title{
Oxidative stress-induced cell cycle blockage and a protease-independent programmed cell death in microaerophilic Giardia lamblia
}

\author{
Esha Ghosh' \\ Arjun Ghosh' \\ Amar Nath Ghosh ${ }^{2}$ \\ Tomoyoshi Nozaki \\ Sandipan Ganguly \\ 'Division of Parasitology; ${ }^{2}$ Division \\ of Electron Microscopy, National \\ Institute of Cholera and Enteric \\ Diseases, Beliaghata, Kolkata, \\ West Bengal, India; ${ }^{3}$ Division \\ of Parasitology, National Institute \\ of Infectious Diseases, Tokyo, Japan
}

Correspondence: Sandipan Ganguly Division of Parasitology, National Institute of Cholera and Enteric Diseases, P 33 CIT Road, Scheme XM, Beliaghata, Kolkata - 7000I0,West Bengal, India

$\mathrm{Tel}+913323633855$

Fax +91 3323705066

Email sandipanganguly@gmail.com

\begin{abstract}
Giardia lamblia is a microaerophilic human gastrointestinal parasite and considered as an early-diverged eukaryote. In vitro oxidative stress generation plays a significant role in cell cycle progression and cell death of this parasite. In the present study hydrogen peroxide, metronidazole, and a modified growth medium without cysteine and ascorbic acid have been chosen as oxidative stress-inducing agents. Cell cycle progression has been found to be regulated by different types of oxidative stresses. Apoptosis is not an established pathway in Giardia, which is devoid of ideal mitochondria, but in the present investigation, apoptosis-like programmed cell death has been found by the experiments like AnnexinV-FITC assay, DNA fragmentation pattern, etc. On the contrary, Caspase- 9 assay, which confirms the caspase-mediated apoptotic pathway, has been found to be negative in all the stress conditions. Protease inhibitor assay confirmed that, even in absence of any proteases, programmed cell death does occur in this primitive eukaryote. All these results signify a novel pathway of programmed suicidal death in Giardia lamblia under oxidative stress. This is the first demonstration of protease-independent programmed cell death regulation in Giardia exclusive for its own specialties.
\end{abstract}

Keywords: Giardia lamblia, oxidative stress, reactive oxygen species, programmed cell death, apoptosis, early branching eukaryotes

\section{Introduction}

Living aerobic organisms, from prokaryotes to complex eukaryotes, have developed elaborate sequences of adaptive mechanisms to maintain oxygen homeostasis and equilibrium. ${ }^{1}$ Any deviation from homeostasis, or physiological change in oxygen pressure, is recognized as an exposure to oxidative stress. ${ }^{2}$ Giardia lamblia is an intestinal protozoan parasite causing malabsorption and diarrhea (giardiasis) in human. It is an early diverging eukaryote with unicellular, binucleated, flagellated structure, without ideal mitochondria, golgi complex or peroxisomes. ${ }^{3,4}$ Measurement of $\mathrm{O}_{2}$ consumption as a function of dissolved $\mathrm{O}_{2}$ indicates that at low levels $(0-50 \mu \mathrm{M})$, the organism is capable of scavenging $\mathrm{O}_{2}$ (apparent $K_{\mathrm{m}}$ for $\mathrm{O}_{2} 6.4 \mu \mathrm{M}$ for the trophozoite). On the contrary, in human gut cell lining, Giardia resides in a higher oxygen tension where the $\mathrm{O}_{2}$ concentration has been measured at $60 \mu \mathrm{M} .{ }^{5}$ This fact has become more interesting as the conventional enzymes for detoxifying reactive oxygen species (ROS) (viz. superoxide dismutase [SOD], catalase, peroxidase, glutathione reductase) are not found in Giardia ${ }^{6}$ Change in the oxygen environment has played the key role in the evolution of aerobic organisms from anaerobes. In case of Giardia, it is also evident that change in oxygen pressure has achieved an important role in its survival strategy as well as the evolution and adaptation of this parasite.

In the present study, three conditions have been chosen to generate oxidative stress in the trophozoites in vitro. First, hydrogen peroxide $\left(\mathrm{H}_{2} \mathrm{O}_{2}\right)$ is a very well known 
chemical reagent that can generate free oxygen radicals very easily. ${ }^{7}$ Secondly, metronidazole is a commonly used drug against giardiasis in the most developing countries. Its mode of action involves free nitro radical generation within the organism. ${ }^{8}$ Third one is a modified medium that is devoid of cysteine and ascorbic acid. Cysteine has been found to protect $G$. lamblia trophozoites from thiol-blocking reactants, indicating a role of reducing agent for protection of crucial thiol groups. Ascorbic acid also protects the trophozoites under high $\mathrm{pO}_{2} .{ }^{9}$ These three conditions would give us an idea about the effects of different modes of oxidative stresses on Giardia cells. Cell cycle analysis using flow cytometer has been carried out to check the effects of oxidative stress on survival of parasitic cells. The changes in whole cell morphology have been studied in each case using transmission electron microscopy (TEM). As mitochondria regulates the caspase-dependent apoptotic cell death pathway in higher eukaryotes and no ideal mitochondria has been reported in Giardia, it was our endeavor to identify whether a programmed cell death (PCD) is possible in this early divergent eukaryote. Several cell death assays have been performed to verify whether the mode of death is apoptotic or necrotic. Protease inhibitor assay has also been performed to check if other proteases like serine/cysteine proteases, calpains, fragmentin/granenzymes, etc are playing any role in parasitic death as they are reported in higher eukaryotes. ${ }^{10}$

Oxygen concentration always plays a vital role in the survival strategy and commitment of death from lower groups of organisms to higher eukaryotes. With the changes in global oxygen concentration from one era to another along thousands of years, lives on earth have evolved themselves from obligatory anaerobes to obligatory aerobic system via several facultative and mutual adaptations. Currently, the detail mechanism is not clear on how these severe changes occurred gradually with time. Studies on oxidative stress regulation in Giardia and similar early branching eukaryotes (EBE) may shed some new ideas about these changes.

\section{Materials and methods Giardia culture maintenance}

Giardia lamblia Portland I was maintained axenically in TYIS-33 medium at $35.5^{\circ} \mathrm{C}$ and sub-cultured at each $48 \mathrm{~h}$.

\section{Standardization of the conditions generating oxidative stress}

Dose and time kinetics of the three different oxidative stressgenerating conditions (as described above) have been standardized following the $\mathrm{IC}_{50}$ values as reported previously.,11
Finally from the standardized data, $0.1 \mu \mathrm{M} \mathrm{H}_{2} \mathrm{O}_{2}$ and $1 \mu \mathrm{g} / \mathrm{ml}$ metronidazole have been administered in all the experiments mentioned below.

\section{Cell fixation and staining for cell cycle analysis in flow cytometry}

Two $\times 10^{6}$ cells were harvested from each culture sets (control and three experimental sets) and the cell pellet were taken by centrifugation at $2000 \mathrm{rpm}$ for $10 \mathrm{~min}$. Cells were washed with phosphate buffered saline (PBS) and treated with methanol for fixation and then in $1 \%$ Triton $\mathrm{X}$ for membrane permeablization. RNase treatment was carried out prior to propidium iodide (PI) staining of the cells. It was incubated on ice and then used for cell cycle analysis using flow cytometer (FC 500; Beckman Coulter, Miami, FL, USA).

\section{Cell death assays}

Cell morphology study by electron microscopy

Control and all sets of experimental samples were harvested $\left(\sim 10^{6}\right.$ trophozoites in each case), prefixed with $3 \%$ gluteraldehyde in $0.1 \mathrm{M}$ sodium cacodylate buffer ( $\mathrm{pH} 7.4$ ) and post fixed in $1 \%$ osmium tetraoxide in $0.1 \mathrm{M}$ sodium cacodylate buffer ( $\mathrm{pH} 7.4$ ). The post-fixed cells were block stained in $1 \%$ uranyl acetate and then treated with graded series of ethanol and subsequently embedded in agar 100 resin (Agar Aids, Stansted, UK). Ultra thin sections were cut in a Leica Ultracut UCT ultramicrotome and stained with uranyl acetate and lead citrate. Sections were examined in a Tecnai 12 Bio-twin transmission electron microscope (FEI, Hillsboro, OR, USA).

\section{Annexin V-FITC assay}

This experiment was performed using Annexin V-FITC Kit (Beckman Coulter), following the manufacturer's protocol. Briefly, cell samples (Control cell as well as oxidative stressed cells) were washed with chilled PBS and cell pellet was resuspended in $1 \times$ binding buffer and kept on ice. Annexin V-FITC solution was added and incubated on ice in dark. Cell preparation was analyzed using confocal microscope (LSM 510, Meta; Carl Zeiss, Thornwood, NY, USA).

\section{FITC-PI staining}

For this experiment the cells were similarly treated with Annexin V-FITC solution (Beckman Coulter) as per manufacturer's protocol as mentioned above. Along with that, PI solution was also added and incubated on ice in the dark. This cell preparation was analyzed for its immunofluorescence using confocal microscope and flow cytometer. 


\section{DNA fragmentation}

Permeabilized cells (as prepared above) were treated with PI to observe the DNA damage in nucleus under confocal microscope.

\section{DNA laddering (degradation)}

Control cells and stressed cells were harvested and resuspended in digestion buffer (10 mM EDTA, $50 \mathrm{mM}$ Tris, $0.5 \%$ SDS Sarcosine, $\mathrm{pH}=8.0$ ) containing $0.5 \mathrm{mg} / \mathrm{ml}$ proteinase K. Samples were incubated at $37{ }^{\circ} \mathrm{C}$ for $1 \mathrm{~h}$ after adding DNase-free RNase $(0.1 \mathrm{mg} / \mathrm{ml})$. After phenol-chloroform treatment, salt preciptation and $70 \%$ ethanol wash, pellet was air dried and resuspended in autoclaved triple distilled water and checked in a $1 \%$ agarose gel stained with ethidium bromide.

\section{Caspase- 9 assay (Colorimetric)}

This assay was performed using Caspase- 9 Assay Kit (Calbiochem ${ }^{\circledR}$, San Diego, CA, USA) following manufacturer's protocol. Briefly, all the control and stressed cell pellets were resuspended in cell lysis buffer provided with the kit and incubated on ice for 10 mins. It was then centrifuged and the supernatant (cytosolic extract) was collected in a tube, kept on ice. After measuring protein concentration it was dissolved in $50 \mu \mathrm{l}$ cell lysis buffer. Then reaction buffer with $10 \mathrm{mM}$ DTT was added to each sample. Finally LEHD-pNA substrate $(200 \mu \mathrm{M}$ final concentration) was added, incubated at $37^{\circ} \mathrm{C}$ and samples were read at $400-405 \mathrm{~nm}$.

\section{Protease inhibitor assay}

Mixture of several protease inhibitors (a broad range protease inhibitor; Roche, Nutley, NJ, USA) were added to the culture and incubated for $1 \mathrm{~h}$. Small water soluble molecules of protease inhibitors were absorbed by the cells along with medium. Then the cells were treated with oxidative stress producing agents. Annexin V-FITC assay and DNAladdering assay were repeated to check if programmed cell death occurred in absence of proteases.

\section{Results and discussion \\ Cell cycle analysis in flow cytometry}

In case of Giardia, normal trophozoites of G1 phase contain $4 \mathrm{~N}$ chromosome. ${ }^{12}$ Hence, in control set, maximum numbers of cells lie in G0/G1 phase (Figure 1i). In case of metronidazole, as observed in flow cytometer, the cell cycle was arrested near S-phase (Figure 1ii). Metronidazole is known to exert its toxic effect through unstable intermediate products (nitro and nitroso-free radicals). ${ }^{14}$ The reactive products also interact with DNA, causing DNA damage, strand breakages, and cross-linking in the nucleic acid and the release of nucleotide phosphates. Hence in our study, the growth arrest of trophozoites near S-phase of cell cycle is due to a similar action of metronidazole. It probably inhibits the DNA segregation and hence prevents the completion of cell division. Peroxides have been found to induce a G1 checkpoint response (Figure 1iii). It suppresses $\mathrm{S}$ phase entry by inhibiting cyclin $\mathrm{E} / \mathrm{Cdk} 2$ activity in a dose-dependent manner. ${ }^{13}$ In this experiment, we have also found that the maximum number of cells lie in sub-G1 stage. It might also be an indication of apoptotic cell death due to peroxide application. For cystein-ascorbate deprived modified medium, most of the cells move to apoptotic death, but a significant amount has a tendency to increase their DNA content (Figure 1iv).
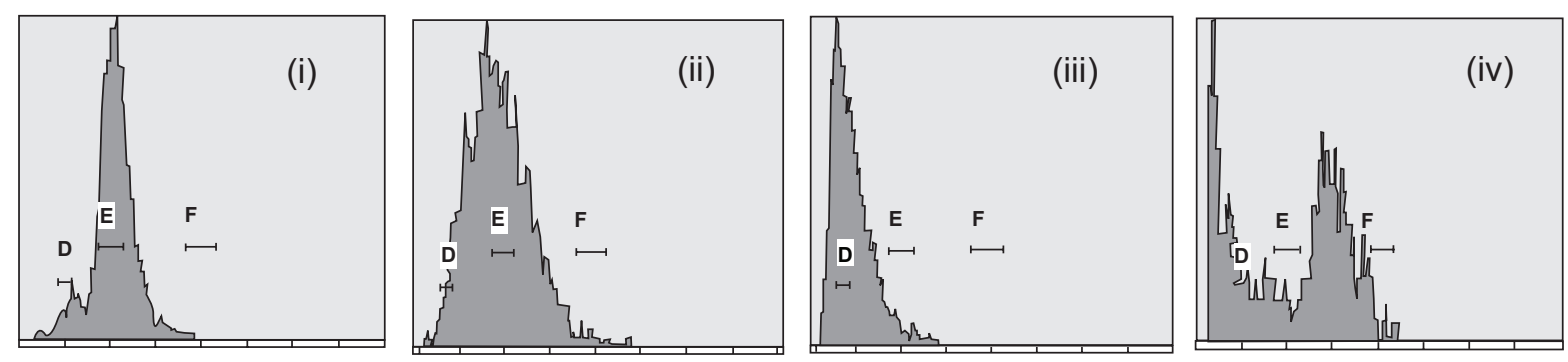

Figure I Cell cycle analysis of control and treated cells. D, E, and F are showing sub-GI*, GI and G2/M phase in the trophozoite cell cycle. Each unit distance in the X-axis denotes 2N DNA content of the trophozoites. Normal Giardia trophozoite contains $4 \mathrm{~N}$ genomic DNA content in GI phase. i) In the control set, maximum no. of trophozoites are showing 4N DNA content with few sub GI population indicating apoptotic cell death. ii) Metronidazole treated cells stop their cell cycle propagation just at synthesis phase and commit apoptotic type of cell death. Cells lie in between $\mathrm{D}$ to late E region. iii) Effect of $\mathrm{H}_{2} \mathrm{O}_{2}$ on Giardia trophozoites is very prompt. All the trophozoites undergo apoptotic type of death and lie sharply in the D region, sharply within sub GI stage. iv) Cells treated with modified medium show a group of trophozoites in D region with sub-GI phase, but few cells are moving beyond S phase towards G2, indicating a tendency to encystation in stressed condition.

Notes: "Sub-GI implies that the DNA content is less than usual GI stage ( $4 \mathrm{~N}$ in case of Giardia). In the dead cells when nuclei start degradation then another peak arises in flow cytometer just left of the original GI peak, indicating that the DNA content is decreasing. 


\section{Cell death assays}

Cell morphology study by electron microscopy

Oxidative stress induced cells have shown apoptotic features like membrane blebbing and increased vacuolation in comparison to control cells under transmission electron microscopy (Figure 2).

\section{Annexin V-FITC assay}

Externalization of phosphatidyl serine (PS) is one of the indicators of apoptosis in the eukaryotic cells. Fluorescein isothiocyanate (FITC)-tagged annexin can bind with the PS when it comes outside the membrane and the fluorescence signal can be measured in a fluorescent microscope. A characteristic green ring formation can be observed in the membrane of an ideal apoptotic cell. In our study we have used this assay to check the mode of death in stress-induced cells. Normal live cells do not show any ring formation where as in case of all the experimental sets treated with different oxidative stress producing agents revealed a clear ring formation in their outer membrane part (Figures 3A, B, D, and F) confirming the mode of cell death to be apoptotic.

\section{FITC-PI staining}

Cells stained with FITC and PI can be targeted for observing their viability and mode of death in a flow cytometer. The gated region in the flow cytometer denotes the type of cells according to their location in the four coordinates. Live cells do not take the stains and lie in the left hand bottom panel where as apoptotic cell population taking FITC stain lies in right hand bottom panel. All the secondary necrotic cells taking the PI stain lie in the right hand upper panel. In our experiment, control live cells did not take any type of stains and consequently lied in the left hand bottom panel (Figure 4A). All the experimental sets showing cell populations in the right hand bottom panel indicate the intake of FITC stain specific for apoptotic death (Figures 4B, C, and D).

\section{DNA degradation}

Cell nucleus takes the PI stain as this dye intercalates in the DNA. In normal live cells, the two nucleus of Giardia can be easily identified after PI staining (Figure 5A). DNA degradation is another hallmark for apoptotic type of cell death. Hence, after the onset of apoptosis the genetic material gets dispersed throughout the cell and dispersed red patches of the dye can be visualized under fluorescent microscope. In all the experimental sets treated with different stressproducing agents, DNA degradation has been observed (Figures 5B, D, and F).

\section{DNA laddering (fragmentation)}

Cells undergoing apoptosis show characteristic morphological and biochemical features. The biochemical marker

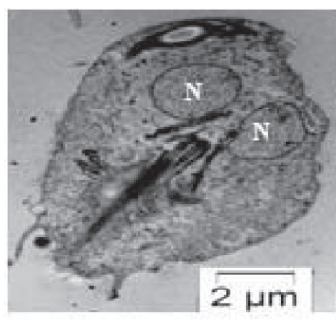

A

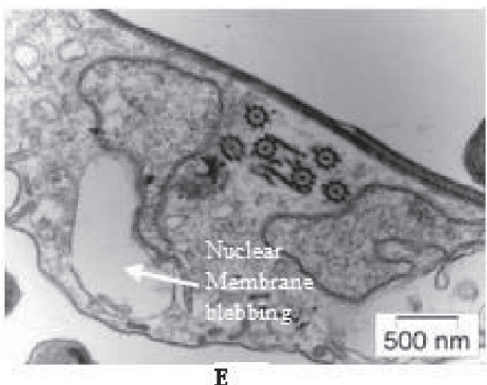

$\mathbf{E}$

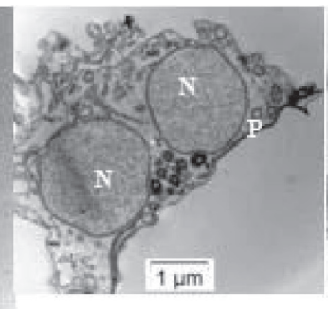

B

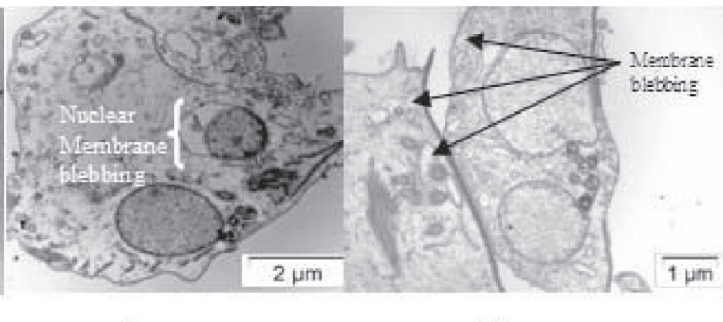

C

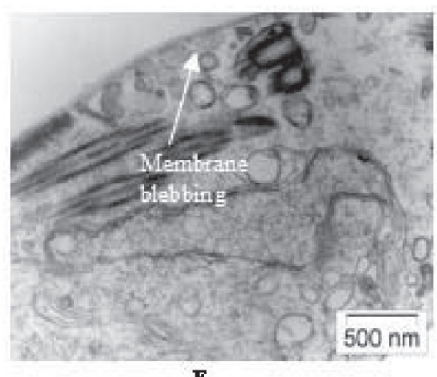

F

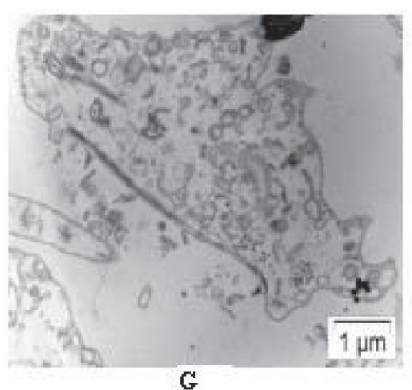

G

Figure 2 Electron microscopic view of control and treated cells. A) Live Giardia cell with two nuclei and flagella. B) Live Giardia (mainly two nuclei are focused). C) Giardia cells treated with $\mathrm{H}_{2} \mathrm{O}_{2}$ : Nuclear membrane blebbing due to PCD. D) Giardia cells treated with metronidazole: Increased vacuolation and outer membrane blebbing due to PCD. E and F) Giardia cells treated with modified medium: Increased vacuolation and nuclear membrane and outer membrane blebbing due to PCD. G) Change in cell morphology after treated with modified medium.

Abbreviations: $\mathrm{N}$, nucleus; $\mathrm{P}$, plasma membrane. 

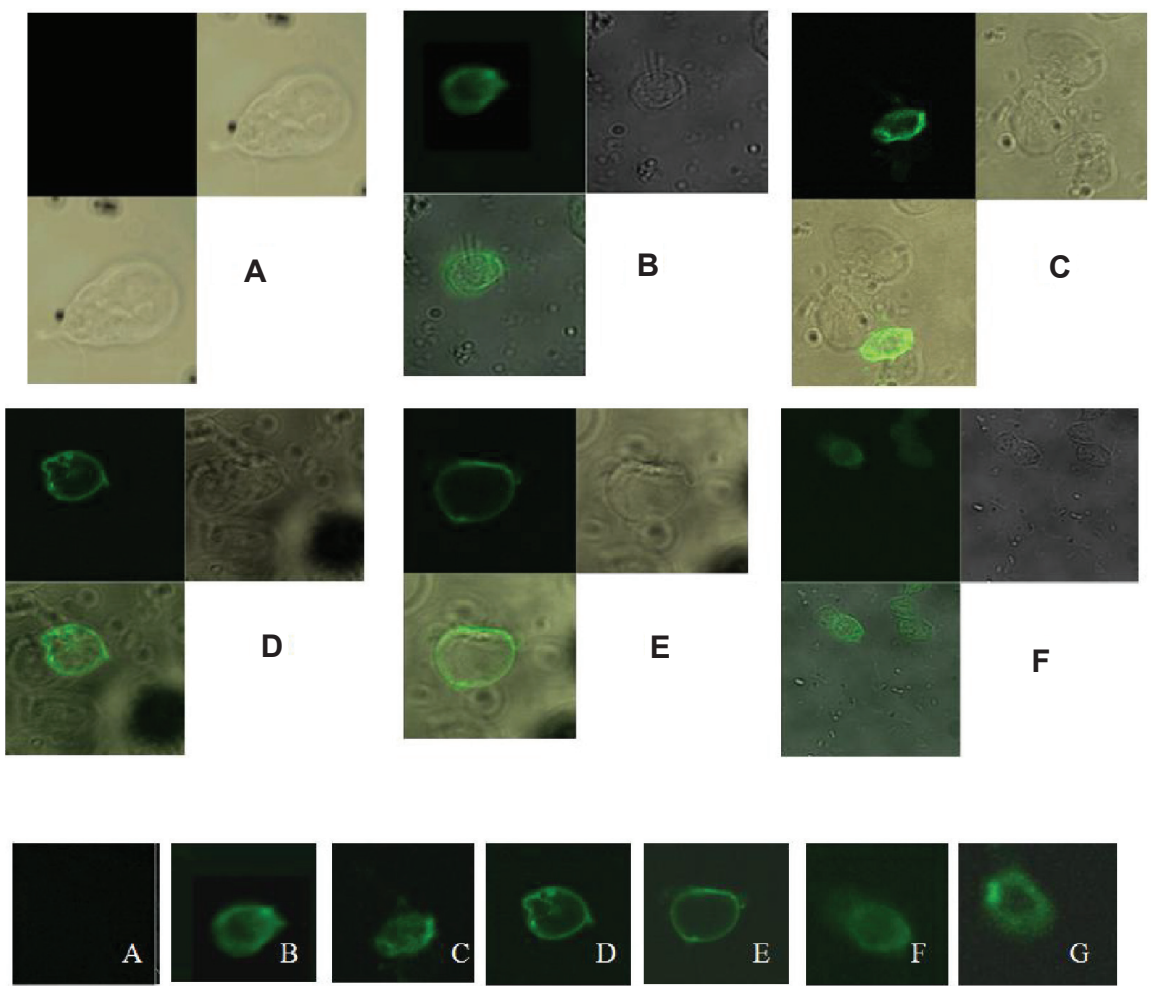

Figure 3 Annexin V-FITC assay. A) normal live Giardia cells, B) Giardia cells treated with $\mathrm{H}_{2} \mathrm{O}_{2}$ for 2 h. C) Giardia cells treated with protease inhibitor cocktail and then further treated with $\mathrm{H}_{2} \mathrm{O}_{2}$ for 2 h. D) Giardia cells treated with metronidazole for 4 h. E) Giardia cells treated with protease inhibitor cocktail and then further treated with metronidazole for 4 h. F) Giardia cells grown in modified medium deprived of Cys-ascorbate for 6 h. G) Giardia cells grown in modified medium deprived of Cys-ascorbate with protease inhibitor cocktail. Panels B to $\mathrm{G}$ clearly show ring formation due to apoptosis.

[E] FL1 Log/FL2 Log - ADC

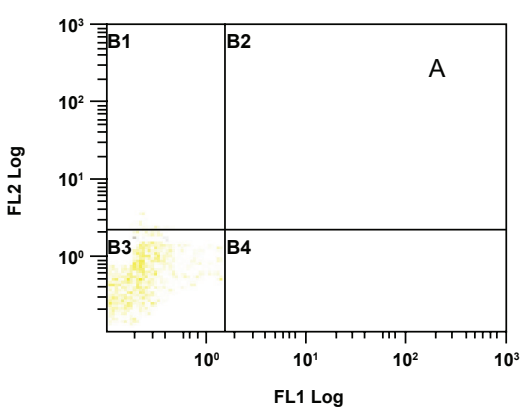

[E] FL1 Log/FL2 Log - ADC

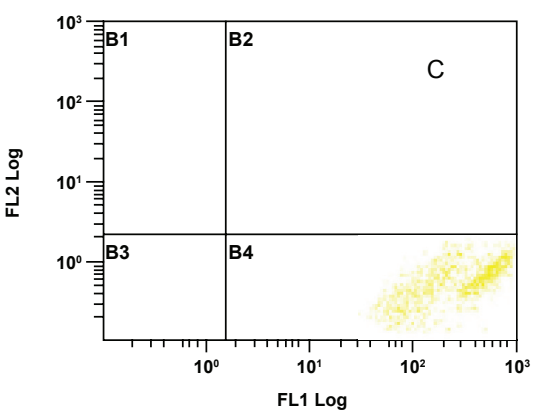

[E] FL1 Log/FL2 Log - ADC

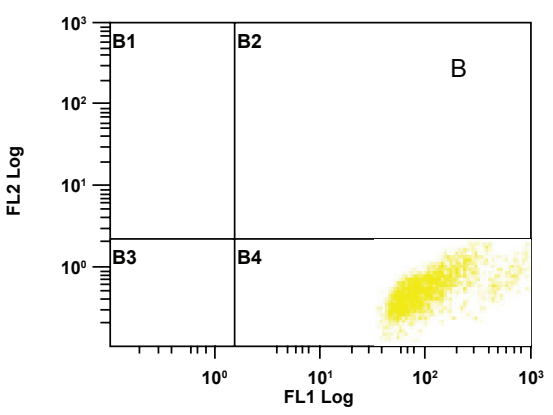

[E] FL1 Log/FL2 Log - ADC

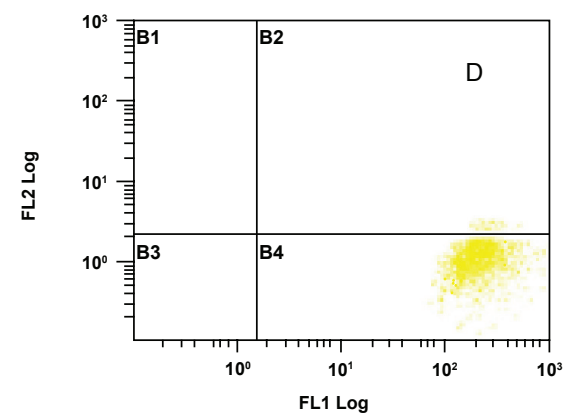

Figure 4 Flow cytometric analysis of FITC-PI stained cells. A) Control live cells lying in the left hand bottom quadrant (B3) as the cell population has not taken any of the two dyes, FITC or PI. B) $\mathrm{H}_{2} \mathrm{O}_{2}$ treated cells after one hour takes FITC and lie in the right hand bottom quadrant (B4) indicating apoptotic type of death. Similarly, in C) and D) cells treated with metronidazole and modified medium respectively, show their mode of death in apoptotic way. 
for apoptosis is the degradation of the genomic DNA, an irreversible event for the programmed cell death and occurs before changes in plasma membrane permeability. DNA fragmentation (mainly in lower molecular weight region) is the characteristic for apoptosis in some lower eukaryotes. Distinct DNA laddering is absent in these organisms. Compared with the control one, all the test samples are showing sheared fragmented DNA in low molecular weight region (Figure 6).

\section{Colorimetric caspase- 9 assay}

Higher eukaryotes having developed apoptotic machinery undergo caspase dependent pathway to carry out apoptosis. Caspase 9 initiates the signaling pathway. It is expected that in a programmed cell death, colorimetric caspase- 9 assay will produce a positive result confirming caspasedependent apoptotic pathway. In our study, a negative result was obtained (Table 1) indicating that the programmed cell death in this parasite is devoid of any caspase-signaling pathway.

\section{Protease inhibitor assay}

In some cases where caspase is not activated in programmed cell death, other proteases such as serine/cysteine proteases, calpeins, or granenzymes, etc get involved in the pathway. So, protease inhibitor assay was performed to check whether other proteases except caspase control the programmed cell death in Giardia. The cells were treated
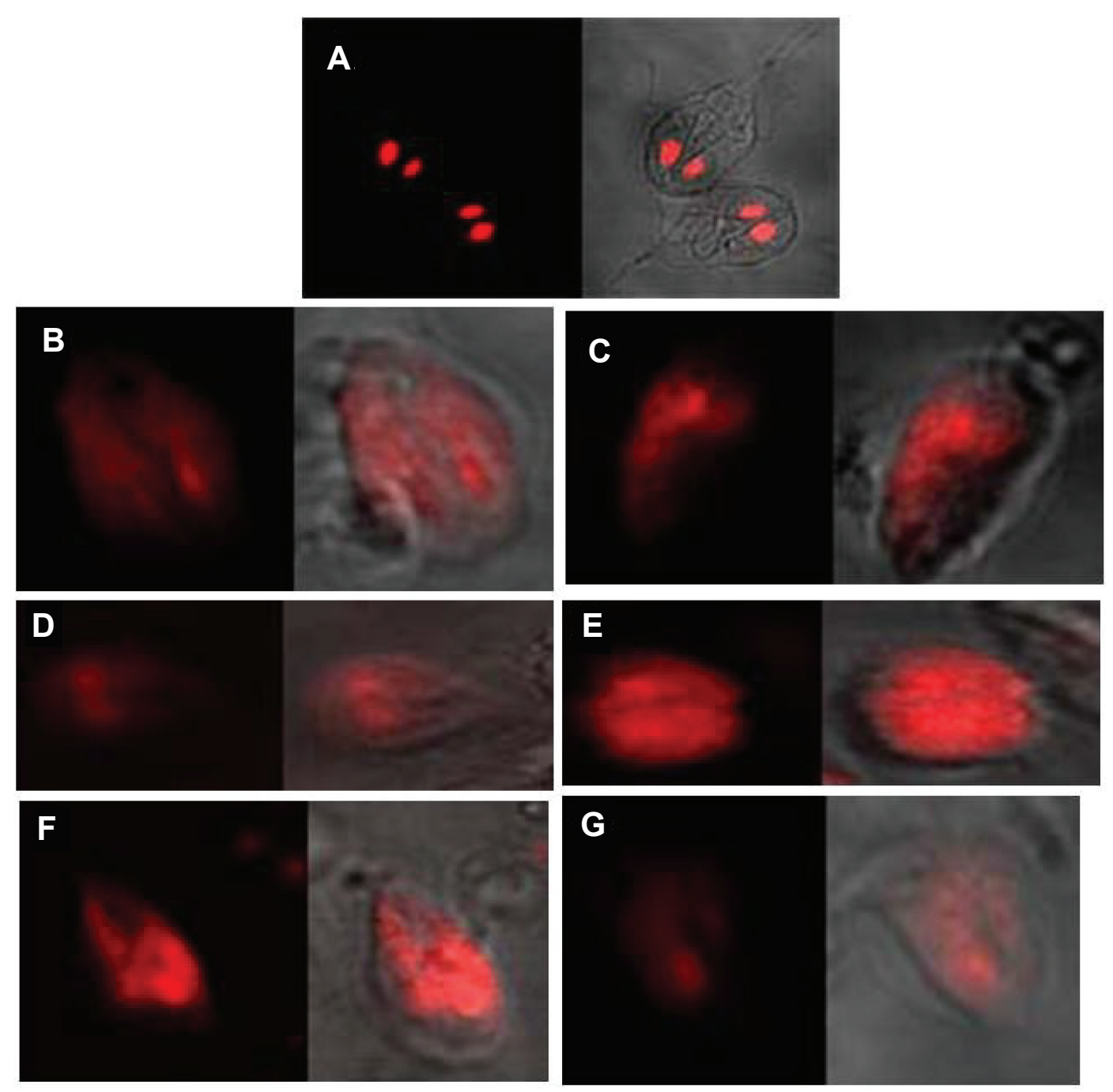

Figure 5 Degradation of nuclei due to programmed cell death in Giardia. A) normal Giardia cells Pl stained, B) Giardia cells treated with $\mathrm{H}_{2} \mathrm{O}_{2}$ for 2 h and PI stained. C) Giardia cells treated with protease inhibitor cocktail and then further treated with $\mathrm{H}_{2} \mathrm{O}_{2}$ for $2 \mathrm{~h}$ and PI stained. D) Giardia cells treated with metronidazole for $4 \mathrm{~h}$ and PI stained. E) Giardia cells treated with protease inhibitor cocktail and then further treated with metronidazole for $4 \mathrm{~h}$ and PI stained. F) Giardia cells grown in modified medium deprived of Cys-ascorbate for $4 \mathrm{~h}$ and PI stained. G) Giardia cells grown in modified medium deprived of Cys-ascorbate with protease inhibitor cocktail and PI stained. Panels B to G clearly show degradation of nuclear content. The phase images in all seven pictures are shown in the right side of the individual pictures. 


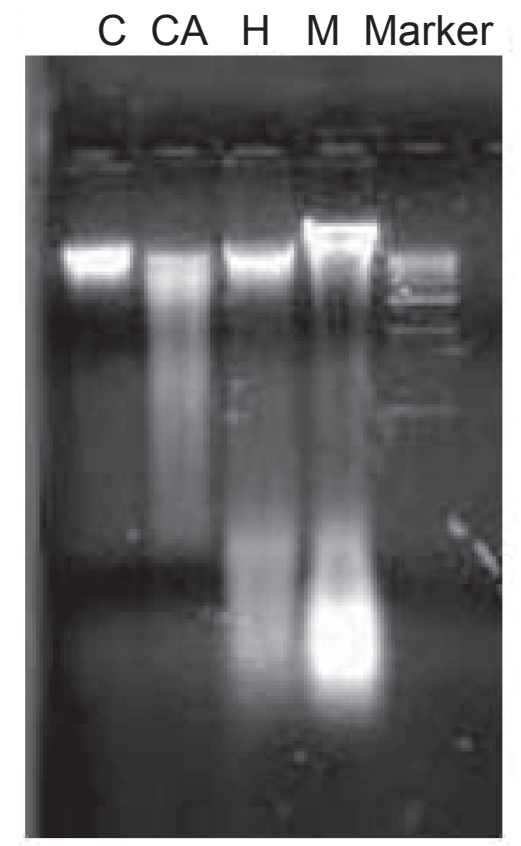

Figure 6 DNA fragmentation assay. Ist panel (C) from left is indicating the DNA from the control live cells. A distinct DNA band can be observed here. In the 2nd panel (CA) fragmented sheared DNA (mainly in lower molecular weight region) was observed in case of modified medium. Similarly in the third and fourth panel $(\mathrm{H}$ and $M)$ for hydrogen peroxide and metronidazole respectively, DNA fragmentation can be observed. The DNA marker ranges from $1-10 \mathrm{~kb}$.

with a large-scale protease inhibitor cocktail before applying the stress-producing agents. Surprisingly, it was found that in absence of proteases, the trophozoites still could undergo PCD. The result has been verified by Annexin V assay (Figures 3C, E, and G) and DNA degradation assay (Figures 5C, E, and G). Characteristic green fluorescent ring formation and scattered PI staining were obtained in each set, respectively.

Table I Result of caspase-9 assay: Higher eukaryotic cell known to undergo caspase-dependent apoptosis has been taken as positive control. On the contrary, living Giardia cells have served as negative control. Giardia dead cells treated with oxidative stress-producing agents have also exhibited negative results

\begin{tabular}{ll}
\hline Sample & $\begin{array}{l}\text { Spectrophotometric } \\
\text { reading at } \mathbf{4 0 5} \mathbf{~ n m}\end{array}$ \\
\hline $\begin{array}{l}\text { Eukaryotic cells undergoing apoptosis } \\
\text { (positive control) }\end{array}$ & 0.516 \\
Giardia lamblia living cells (negative control) & 0.021 \\
Giardia lamblia cells treated with $\mathrm{H}_{2} \mathrm{O}_{2}$ & 0.013 \\
$\begin{array}{l}\text { Giardia lamblia cells treated with } \\
\text { metronidazole }\end{array}$ & 0.018 \\
$\begin{array}{l}\text { Giardia lamblia cells treated } \\
\text { with modified medium }\end{array}$ & 0.023 \\
\hline
\end{tabular}

A similar type of report has been obtained in the case of Trichomonas vaginalis. ${ }^{15}$ PI has shown typical DNA degradation in the dead cells. But it has been found that caspase or other proteases are not responsible for this type of death. Cells, after incubating with protease inhibitors when undergo stress, still committed death in programmed pathway. This was confirmed by Annexin V test and DNA fragmentation pattern, as before. Two cases in Leishmania and Blastocystis have been reported where also the PCD is independent of caspase pathway or other proteases. ${ }^{16,17}$ It is possible that these EBE including our model organism G. lamblia have undergone PCD using a novel pathway.

\section{Acknowledgments}

This study was supported by a grant from Okayama University Program of Founding Research Centre for Emerging and Reemerging Infectious Disease, Ministry of Education, Culture, Sports, Science and Technology of Japan. The authors would like to thank Prof Y Takeda and Dr GB Nair, NICED, Japan for their constructive comments and suggestions during the study. Authors are also thankful to Debarati Ganguly of Calcutta University for her critical reading and English correction.

\section{References}

1. Acker H. Mechanisms and meaning of cellular oxygen sensing in the organism. Respir Physiol. 1994;95(1):1-10.

2. Sen CK, Packer L. Antioxidant and redox regulation of gene transcription. FASEB J. 1996;10(7):709-720.

3. Adam RD. The biology of Giardia spp. Microbiol Rev. 1991;55(4): 706-732.

4. Adam RD. Biology of Giardia lamblia. Clin Microbiol Rev. 2001;14(3):447-475.

5. Atkinson HJ. Respiration in nematodes. In: Zuckerman BM, editor. Nematodes as Biological Models. New York, NY: Academic Press; 1980. p. 116-142.

6. Brown DM, Upcroft JA, Upcroft P. Free radical detoxification in Giardia duodenalis. Mol Biochem Parasitol. 1995;72(1-2):47-56.

7. Lindley TA, Chakraborty PR, Edlind TD. Heat shock and stress response in Giardia lamblia. Mol Biochem Parasitol. 1988;28:135-144.

8. Brown DM, Upcroft JA, Edwards MR, Upcroft P. Anaerobic bacterial metabolism in the ancient eukaryote Giardia duodenalis. Int J Parasitol. 1998;28(1):149-164.

9. Tekwani BL, Mehlotra RK. Molecular basis of defence against oxidative stress in Entamoeba histolytica and Giardia lamblia. Microbes Infect. 1999;1:385-394.

10. Patel T, Gores GJ, Kaufmann SH. The role of proteases during apoptosis. FASEB J. 1996;10(5):587-597.

11. Sadhu H, Mahajan RC, Ganguly N. Flowcytometric assessment of the effect of drugs on Giardia lamblia trophozoites in vitro. Mol Cell Biochem. 2004;265:151-160.

12. Bernander R, Daniel Palm JE, Svard SG. Genome ploidy in different stages of Giardia lamblia life cycle. Cell Microbiol. 2001; 3(1):55-62.

13. Clopton DA, Saltman P. Low level oxidative stress causes cell cycle specific arrest in cultured cells. Biochem Biophys Res Commun. 1995;210:189-196. 
14. Ellis JE, Cole D, Lloyd D. Influence of oxygen on the fermentative metabolism of metronidazole sensitive and resistant strains of Trichomonas vaginalis. Mol Biochem Parasitol. 1992;56:79-88.

15. Chose O, Sarde CO, Noël C, Gerbod D, Jimenez JC, Brenner C. Cell death in protists without mitochondria. Ann N Y Acad Sci. 2003;1010:121-125.
16. Dutta A, Bandyopadhyay S, Mandal C, Chatterjee M. Aloe vera leaf exudate induces caspase induced cell death in Leishmania donovani promastigotes. J Med Microbiol. 2007;56:629-636.

17. Nasirudeen AM, Tan KS. Programmed cell death in Blastocystis hominis occurs independently of caspase and mitochondrial pathways. Biochimie. 2005;87(6):489-497. 